\title{
Anticariogenic Effect of Selenium Nanoparticles Synthesized Using Brassica oleracea
}

\author{
Ganapathy Dhanraj $\mathbb{D}^{1}$ and Shanmugam Rajeshkumar $\mathbb{D}^{2}$ \\ ${ }^{1}$ Department of Prosthodontics, Saveetha Dental College, Saveetha Institute of Medical and Technical Sciences, \\ Chennai 600 077, India \\ ${ }^{2}$ Department of Pharmacology, Saveetha Dental College, Saveetha Institute of Medical and Technical Sciences,
} Chennai 600 077, India

Correspondence should be addressed to Shanmugam Rajeshkumar; ssrajeshkumar@hotmail.com

Received 20 April 2021; Revised 16 June 2021; Accepted 24 June 2021; Published 10 July 2021

Academic Editor: Abdelwahab Omri

Copyright (C) 2021 Dhanraj Ganapathy and Rajeshkumar Shanmugam. This is an open access article distributed under the Creative Commons Attribution License, which permits unrestricted use, distribution, and reproduction in any medium, provided the original work is properly cited.

\begin{abstract}
Selenium is a trace element in the human body present in various enzymes with antioxidant activities and several functional proteins. This study is aimed at synthesizing selenium nanoparticles using Brassica oleracea (broccoli) and characterizing and assessing the antioxidant and antimicrobial effectiveness against cariogenic microorganisms. UV-visible spectrum displayed a peak at $370 \mathrm{~nm}$ which confirms the formation of SeNPs. TEM images of synthesized selenium nanoparticles showed polydisperse nanoparticles, spherical. The size of the particles ranged from 10 to $25 \mathrm{~nm}$, and the average particle size obtained was $15.2 \pm 1.9 \mathrm{~nm}$. SEM images of nanoparticles were spherical and ranged in size from 10 to $28 \mathrm{~nm}$. The SeNPs showed effective antimicrobial activity against cariogenic pathogens. The SeNPs synthesized with Brassica oleracea extract can be incorporated in toothpaste, gums, and mouthwashes that are cost-effective and biocompatible and used for the prevention of dental caries.
\end{abstract}

\section{Introduction}

Owing to their characteristic attributes, nanoparticles find extensive implementation in several scientific, medicinal, and industrial domains $[1,2]$. Among the various kinds of nanoparticles, selenium has attracted appreciable interest due to its greater bioactivity, protein interactions, strong absorption capability, and reduced toxicity, together with interdisciplinary applications in medicine, therapeutic sciences, nanobioinformatics, and nanobiotechnology [3].

Selenium corresponds to Group VI in the periodic table as an integral trace element. Numerous antioxidant enzymes and functional protein molecules contain selenium and maneuver a crucial role in mitigating oxidative stress, thus minimizing the damage in cardiovascular diseases, cancer, diabetes, and hypercholesterolemia. On the other hand, the intake of selenium is necessary for different metabolic processes and indiscriminate intake beyond acceptable concentration leads to selenium toxicity [4-6].
Selenium is found in nature as ionic forms of selenite $\left(\mathrm{Na}_{2} \mathrm{SeO}_{3}\right)$, selenate $\left(\mathrm{Na}_{2} \mathrm{SeO}_{4}\right)$, and selenium oxide (SeO) among the other forms. Elemental selenium nanoparticles manifest lesser cytotoxicity compared with selenite $\left(\mathrm{SeO}_{3}\right)$ and selenate compounds $\left(\mathrm{SeO}_{4}\right)$ and show anticancer and several therapeutic properties that enable them to be utilized in versatile medicinal applications $[7,8]$. Researchers have reported on the antioxidant function of SeNPs. SeNPs can unswervingly scavenge the free radicals in a size-dependent manner within the size ranging from 5 to $200 \mathrm{~nm}$ [9].

Traditional physiochemical methods of SeNP synthesizing are cumbersome, nonecological, and require prerequisites such as high temperatures, toxic substances, reagents, precursors, and complicated procedures. Besides, in the conventional process, the form, size, and instability of the synthesized particle are the main limitations [10]. This creates a great desire to synthesize nanoparticles with harnessed size and nanometer morphology in an eco-friendly mode [11]. The size and the shape of nanoparticles are permanently 
related to the unique characteristics, work, and application of nanomaterials $[12,13]$. Because of its eco-friendliness, low toxicity along with increased stability, small size, and shape distribution, emerging advances in nanoparticle generation using green syntheses are attracting much attention today $[14,15]$.

Brassica oleracea (broccoli) has all the nutrients that prove its exceptional health benefits, including vitamins, minerals, secondary metabolites, and fiber. The active principles for demonstrating medicinal properties are the breakdown products of sulfur-containing glucosinolates, phenolic isothiocyanates, antioxidants, vitamins, and dietary minerals. These bioactive compounds most likely mediate the medicinal properties of broccoli consumption by the inducement of a range of functions such as antioxidant behavior, enzyme regulation, and the control of apoptosis [16].

Organic chemicals such as S-methyl cysteine sulfoxide and glucosinolates present in Brassica oleracea in conjunction with other ingredients inclusive of vitamins $\mathrm{C}, \mathrm{K}$, and $\mathrm{E}$ and minerals like selenium, iron, and zinc and polyphenols such as kaempferol, glucosides of quercetin, and isorhamnetin are likely to be accountable for the various health benefits of broccoli $[17,18]$.

Concerning dental problems, caries is one of the most common human diseases characterized by susceptibility to enamel and dentin tissue damage due to acidic byproducts generated from dietary carbohydrates owing to bacterial fermentation [19]. This problem is initiated by the acid production from bacteria that ferment carbohydrates resulting in demineralization of dental enamel and the development of caries. Supplementation of calcium and phosphate can aid in the rarefy demineralization of teeth to avoid dental caries. Such ions are also present in the saliva, and their concentration determines remineralization and demineralization [20,21].

Nanocarriers are presently being reasoned for their application in dentistry, and their particular characteristics identify possible use in the delivery of antimicrobial agents in the prevention and management of oral diseases caused by microbial invasion. Implementation of nanomaterials in dentistry may be divided into two major categories: dental preventive and restorative care [22]. According to caries research, novel approaches to prevent and manage dental caries have been implemented through nanotechnology, in particular through plaque-related biofilm regulation and remineralization of carious lesions [23].

In this area, nanotechnological advances have also provided advantages as novel drivers of innovation. It has been shown that natural biomineralization, which is an integral repair mechanism, is induced by the use of nanotechnology. For enhancing oral health through the prevention of dental caries, the use of nanomaterials in toothpaste and other mouth rinsing solutions can be recommended. Caries is often arrested by nanomaterials used in polishing agents and dental restorative materials. Per se, antimicrobial nanoparticles can inhibit the growth of bacteria inducing dental caries [24].

Dental caries can be addressed by nanotechnology in two primary methods. In the first method, fluoride- and calciumreleasing nanomaterials such as calcium fluoride, calcium phosphate, hydroxyapatite, and fluorohydroxyapatite are used in a process called remineralization. The second method is to imbibe antimicrobial nanomaterials such as selenium, platinum, quaternary ammonium compounds, and zinc oxide nanoparticles $[25,26]$. To present better results, a blend of these two methods can also be used. The anticariogenic effects of selenium nanoparticles through inhibition of cariogenic microbes were not studied extensively and thus need to be explored further. Hence, this study was formulated with the aim to green synthesize selenium nanoparticles using Brassica oleracea and study its characterization and antioxidant and antimicrobial effectiveness against cariogenic microorganisms.

\section{Material and Methods}

2.1. Preparation of Broccoli Extract. Brassica oleracea was procured from organic farms in Poonamallee. Broccoli was washed thoroughly under tap water and made into small pieces to shade dry it for 3-4 days. After that, the shadedried broccoli pieces were ground into a fine powder. From that, finely grounded broccoli powder $0.7 \mathrm{~g}$ was taken and added to $70 \mathrm{~mL}$ distilled water. The mixture was kept in a heating mantle at $60-80^{\circ} \mathrm{C}$ for 10 minutes. Then, the boiled mixture was filtered using Whatman No. 1 filter paper. The filtered broccoli extract was stored in the refrigerator for selenium nanoparticle synthesis.

2.2. Preparation of Selenium Nanoparticle. $30 \mathrm{mM}$ of sodium selenite was prepared in $70 \mathrm{~mL}$ distilled water. To that $30 \mathrm{~mL}$ filtered broccoli, the extract was added and kept in a magnetic stirrer for 2-3 days at 650-800 rpm to obtain uniform dispersion, a mandatory condition in nanoparticle synthesis. The color changes in the reaction mixture were noted continuously using a double-beam UV-visible spectrophotometer at the different wavelength range from 250 to $650 \mathrm{~nm}$. The synthesized broccoli extract-mediated selenium nanoparticles were centrifuged at $8000 \mathrm{rpm}$ for 10 minutes. The obtained selenium nanoparticle pellet was powdered using a hot air oven at $70^{\circ} \mathrm{C}$ for 2 hours and preserved in airtight vials for further use.

2.3. Characterization of Selenium Nanoparticles. The maximum absorbance of broccoli-mediated selenium nanoparticles was measured by using a double-beam UV-vis spectrophotometer (UV-2450, Shimadzu) in the wavelength range of $250-650 \mathrm{~nm}$. The morphological features such as size and shape were analyzed by TEM. The functional groups present in the synthesized selenium nanoparticles were identified by using FT-IR analysis and also subjected to test the elemental analysis using an energy-dispersive X-ray detector (EDX) attached to the SEM machine.

2.3.1. Antioxidant Activity. The DPPH assay was used to test the antioxidant activity of biogenic synthesized selenium nanoparticles. Diverse concentrations $(2-10 \mu \mathrm{g} / \mathrm{mL})$ of broccoli extract-interceded selenium nanoparticle were mixed with $1 \mathrm{~mL}$ of $0.1 \mathrm{mM} \mathrm{DPPH}$ in methanol and $450 \mu \mathrm{L}$ of $50 \mathrm{mM}$ Tris $\mathrm{HCl}$ buffer ( $\mathrm{pH}$ 7.4) and incubated for 30 minutes. Later, the reduction in the quantity of DPPH free 


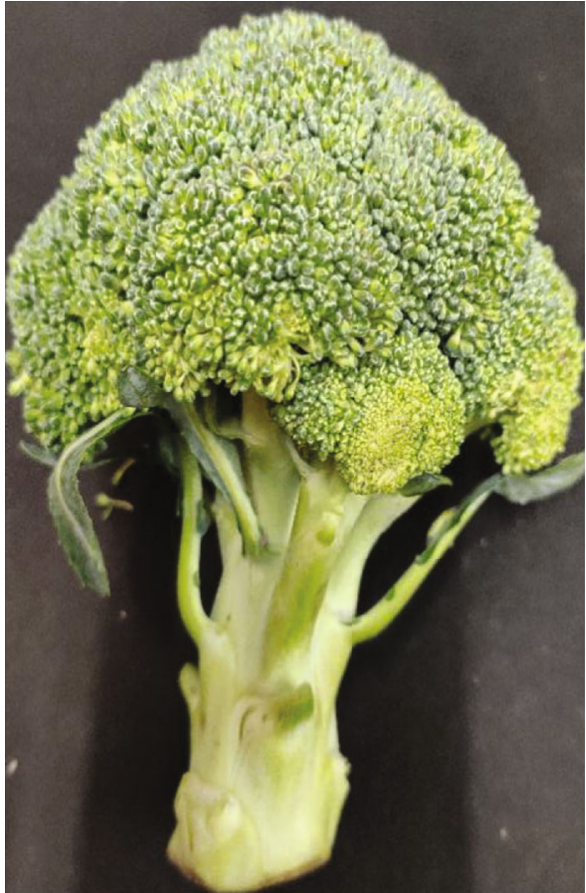

(a)

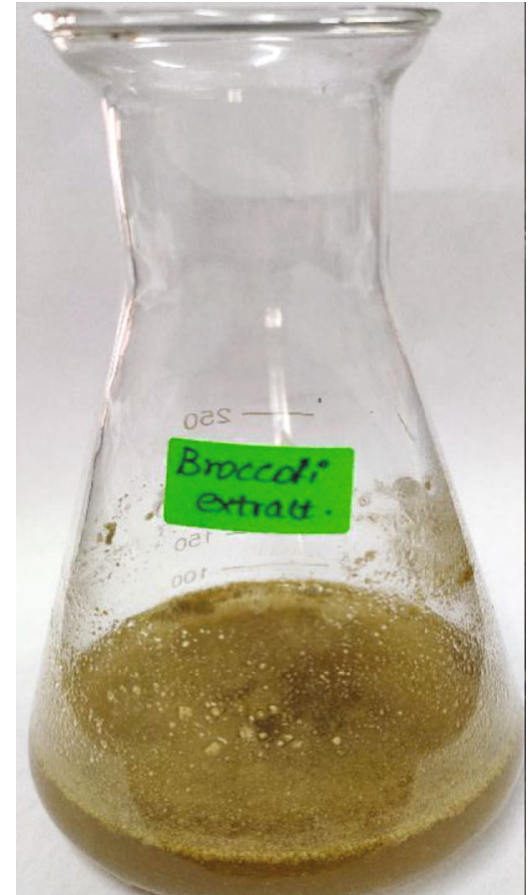

(b)

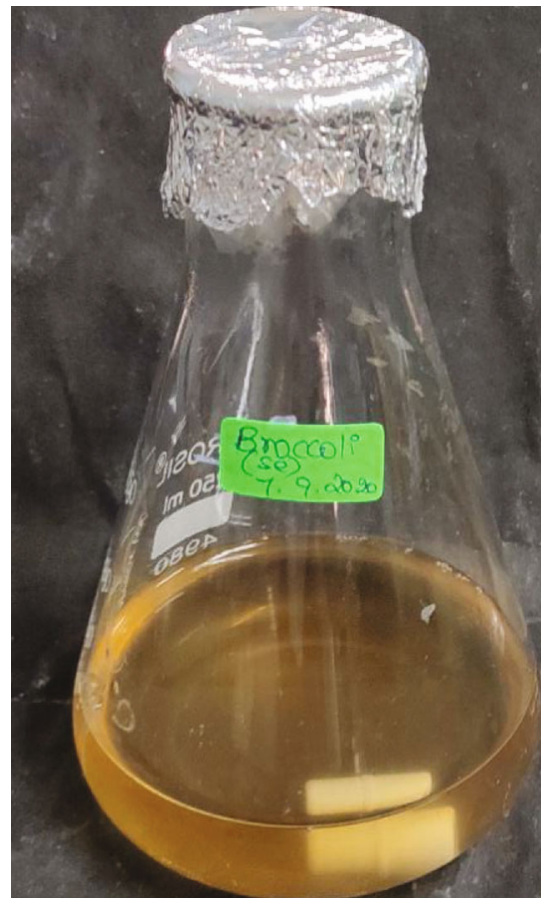

(c)

Figure 1: Color changes on selenium nanoparticles synthesis: (a) broccoli; (b) plant extract; (c) selenium nanoparticles.

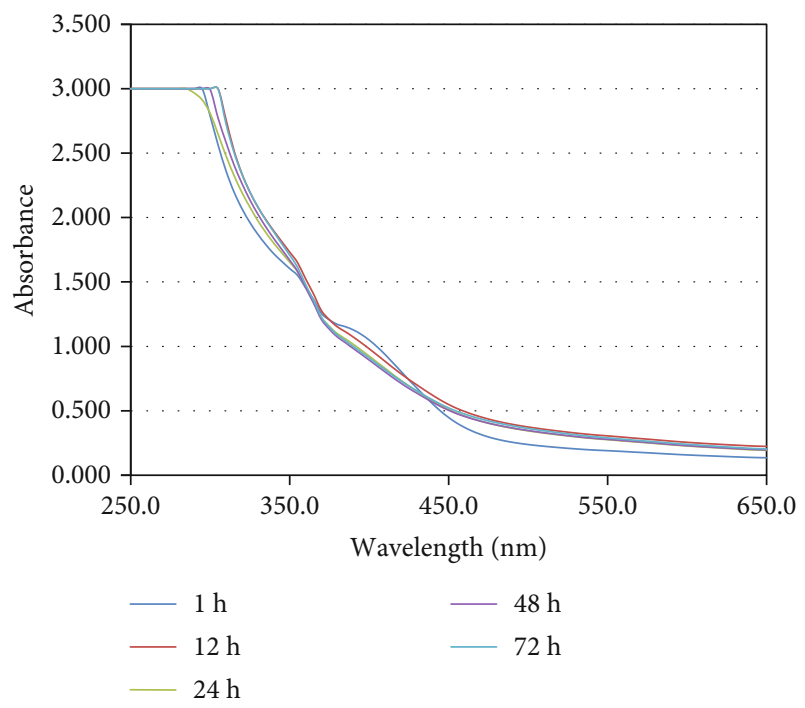

FIGURE 2: UV-vis spectroscopic analysis of broccoli-mediated selenium nanoparticles at different time intervals and wavelength is from $250 \mathrm{~nm}$ to $650 \mathrm{~nm}$.

radicals was assessed depending on the absorbance at $517 \mathrm{~nm}$. BHT was employed as control. The percentage of inhibition was determined from the following equation:

\%inhibition $=\frac{\text { Absorbance of control }- \text { Absorbance of test sample }}{\text { Absorbance of control }} \times 100$.

2.3.2. Anticariogenic Activity. The anticariogenic activity of broccoli-mediated selenium nanoparticles was tested against

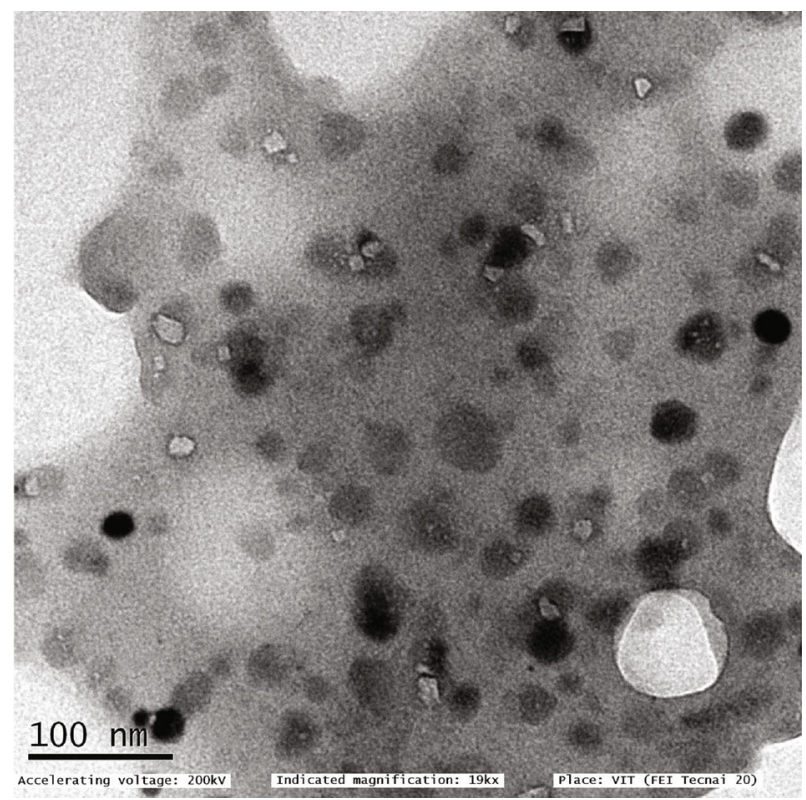

Figure 3: Transmission electron microscopic image of selenium nanoparticles synthesized using broccoli extract and scale measures at $100 \mathrm{~nm}$.

oral pathogens such as S. mutans, S. aureus, E. faecalis, Lactobacillus, and C. albicans. Mueller Hinton agar was utilized for this activity to determine the zone of inhibition. Mueller Hinton agar was prepared and sterilized for 45 minutes at $120 \mathrm{lbs}$. Media were poured into the sterilized plates and let it stabilize for solidification. The wells were cut using the good cutter, and the test organisms were swabbed. The selenium 


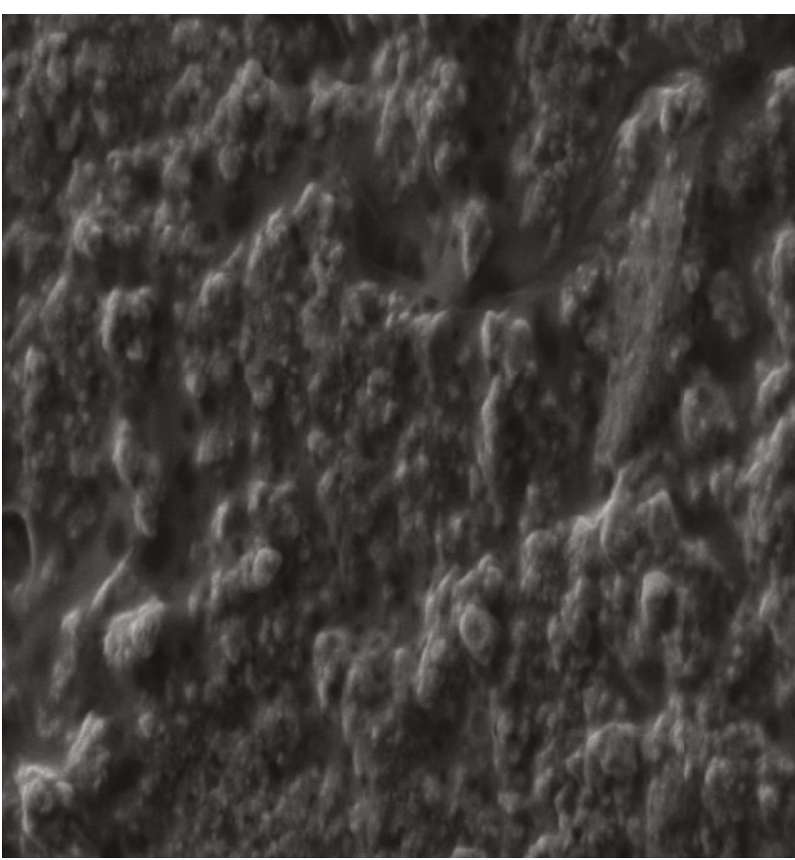

(a)

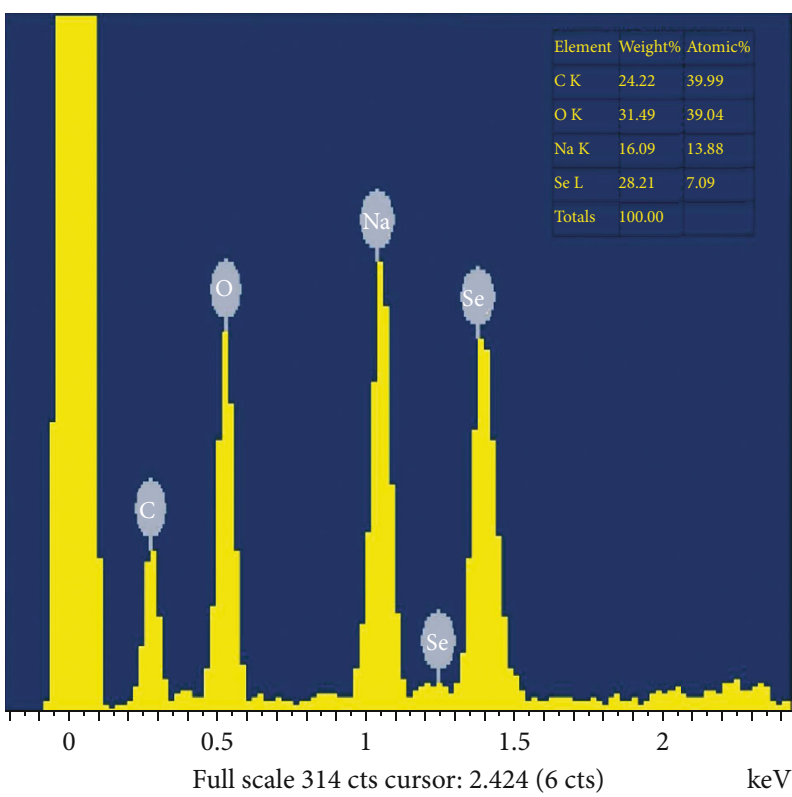

(b)

Figure 4: Selenium nanoparticles: (a) scanning electron microscopy image; (b) elemental X-ray diffraction spectrum.

nanoparticles with different concentrations were loaded, and the plates were incubated at $37^{\circ} \mathrm{C}$ for 24 hours. After the incubation time, the zone of inhibition was measured.

\section{Results and Discussion}

Compared to physical or chemical approaches of nanoparticle synthesis, one of the effective methods is the generation of nanoparticles utilizing green sources such as plant phytocompounds, proteins, and enzymes as reducing agents in the case of microbes. The benefits are that they are nontoxic materials, requiring relatively less complicated and affordable equipment, biodegradable material processing, improved selectivity, and high yields.

Visual coloring is the first stage in the moulding of nanoparticles. It was observed following the incubation period that the color changed to brown. The reaction mixture with Brassica oleracea and sodium selenite during incubation showed a time-dependent color shift of $48 \mathrm{~h}$ at $30^{\circ} \mathrm{C}$, as shown in Figure 1 . At the primary reaction stage, the color of the concoction was yellow, which progressively changed to brown over time. After 48 hours of incubation, no further alteration in color was observed. This brown color may be attributed to the arousal of the surface plasmon vibrations by the selenium nanoparticles and thus render beneficial spectroscopic evidence of their formation.

UV-visible spectra have shown a large peak at $370 \mathrm{~nm}$, supporting the formation of SeNPs [27]. The peak intensity increased over time. No further significant elevation in peak intensity was observed after 48 hours of the reaction. The peak amplitude has increased over time because of the reduction of $\mathrm{SeO}_{3} 2$ - to $\mathrm{Se} 0$. No further peak increase was observed rafter $48 \mathrm{~h}$, suggesting a maximum conversion of $\mathrm{SeO}_{3} 2$ - to $\mathrm{Se} 0$ (Figure 2). Transmission electron microscopy (TEM) images of selenium nanoparticles synthesized in nutrient broth supplementation with $1.0 \mathrm{mM}$ selenite at 72 hours of incubation are shown in Figure 3. The generated nanoparticles were polydisperse, shaped spherically; the particle size ranged from 10 to $25 \mathrm{~nm}$, and the mean particle size that prevailed from the distribution of the corresponding diameter was approximately $15.2 \pm 1.9 \mathrm{~nm}$ (Figure 3 ).

Synthesized plant extracts, such as hydrogen bond and the electrostatic interaction, were present in the SEM image of SeNPs and were mounted on the surface because of the interaction with the bioorganic capping molecules attached to the SeNPs. Synthesized SeNPs with sizes ranging from $10.32 \mathrm{~nm}$ to $25.88 \mathrm{~nm}$ were observed (Figure 4(a)). SEM study of the synthesized SeNPs was easily distinguishable due to their size variation. The SEM picture showed the majority of nanoparticles are spherical in size, ranging from 10 to $25 \mathrm{~nm}$, and uniformly distributed. SEM analysis of SeNPs was readily distinguishable due to the size difference of synthesized SeNPs. Elemental X-ray diffraction assay was used to assess the elemental percentage and presence; selenium nanoparticles synthesized with broccoli extract had an elemental presence of selenium with a 28.02 percent weight percentage and a 7.09 percent atomic percentage (Figure 4(b)).

FT-IR estimation was performed to determine the possible bands present in the biomolecules accountable for near capping peaks and effective stabilization of the metal NPs 


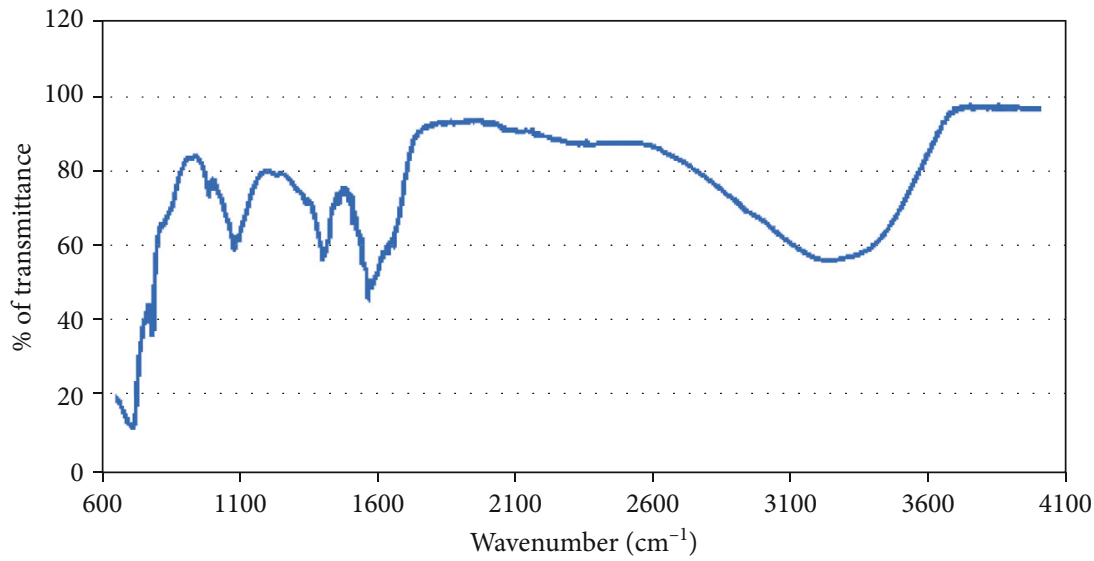

FIGURE 5: Fourier-transform infrared spectroscopy of selenium nanoparticles synthesized using broccoli aqueous extract.

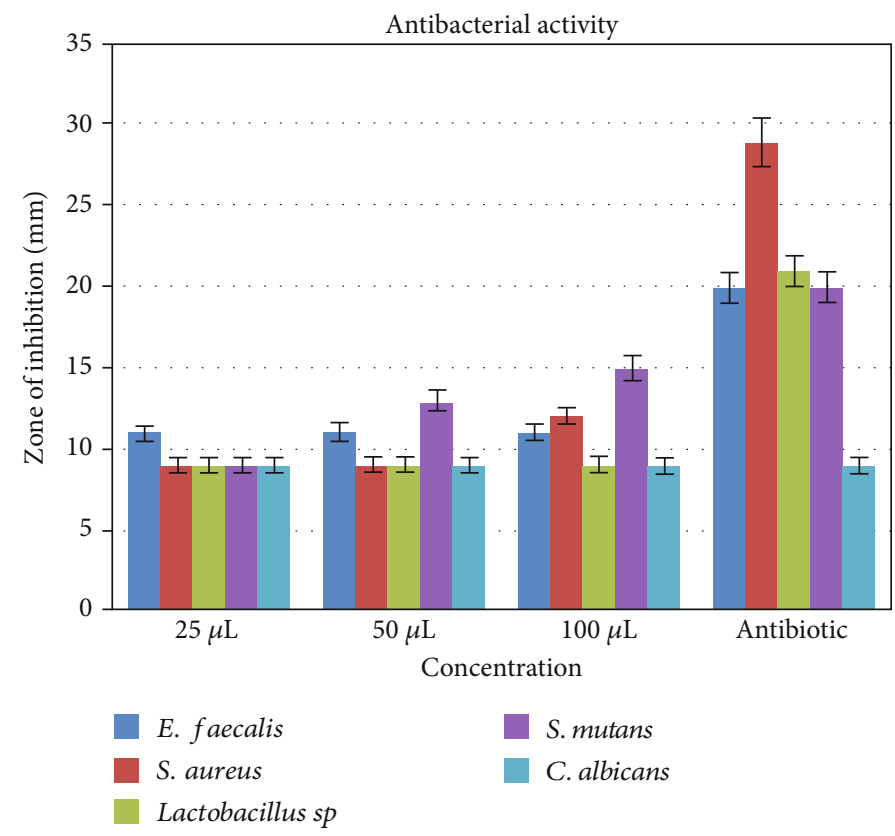

Figure 6: Anticariogenic activity against caries-causing microbes (S. mutans, Lactobacillus sp., E. faecalis, S. aureus, and C. albicans) at different concentrations and zone of inhibition was measured in millimeter.

synthesized by broccoli extract. FT-IR estimation revealed a weak broad peak at $3235.32 \mathrm{~cm}^{-1}$ indicating the presence of alcohol functional group with $\mathrm{O}-\mathrm{H}$ stretching, strong to medium peaks at $1595.30 \mathrm{~cm}^{-1}$ indicating amine group with $\mathrm{N}-\mathrm{H}$ bending, $1407.07 \mathrm{~cm}^{-1}$ indicative of fluoro compounds with C-F stretching, and $1099.56 \mathrm{~cm}^{-1}$ indicating aliphatic ether with $\mathrm{C}-\mathrm{O}$ stretching (Figure 5).

Selenium nanoparticles displayed potent antimicrobial activity against caries-causing microorganisms at all concentrations closely comparable to the antibiotic controls. The maximum antimicrobial activity against $S$. mutans was observed (Figures 6 and 7). Figure 8 shows the antioxidant activity by DPPH was also important and displayed more efficacy than the controls at concentrations of $10 \mu \mathrm{L}$ and $20 \mu \mathrm{L}$ selenium nanoparticles. Selenium has a major biological role in species health and is an important component of the composition of glutathione peroxidases, thioredoxin reductases, and other selenoenzymes [4]. Glutathione peroxidase is an enzyme that prevents tissue oxidative stress and can thus inhibit the activity of harmful free radicals.

UV-vis spectroscopy is one of the most commonly used methods for the structural characterization of SeNPs. The size and shape of the tracked aqueous suspension NPs can usually be analyzed by UV-vis spectroscopy. Our outcome indicates that $C$ leaf extract synthesizes SeNPs. Measurements based on data and spectrophotometers range from 200 to $700 \mathrm{~nm}$ with a $420 \mathrm{~nm}$ peak indicating SeNP performance.

Energy-dispersive microanalysis spectroscopy was carried out using EDX techniques to gain further perception of the SeNPs' functionality. Our results suggest that selenium binding energies have EDaX peaks of approximately 72.64. The outcome indicates the presence in the pure form of SeNPs of 


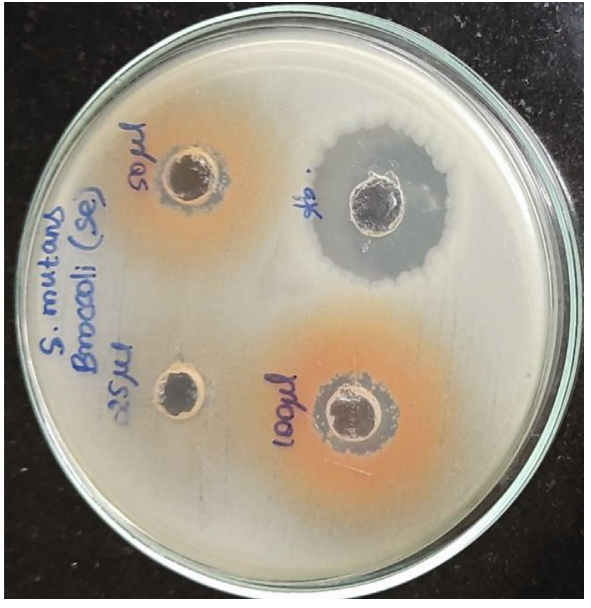

(a)

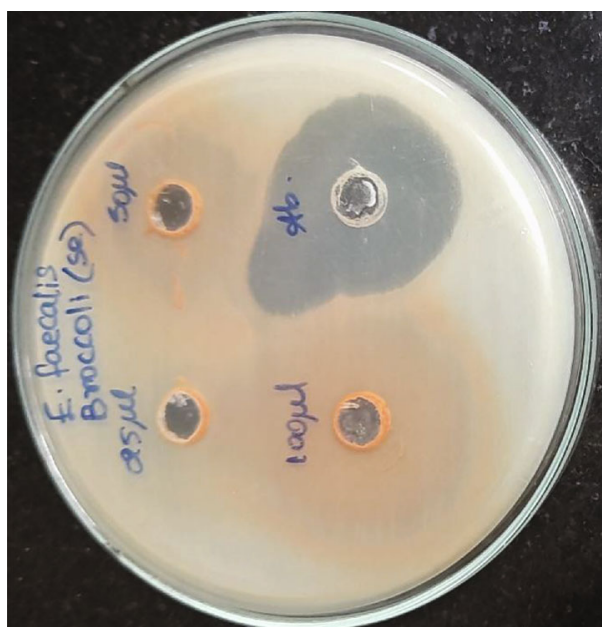

(c)

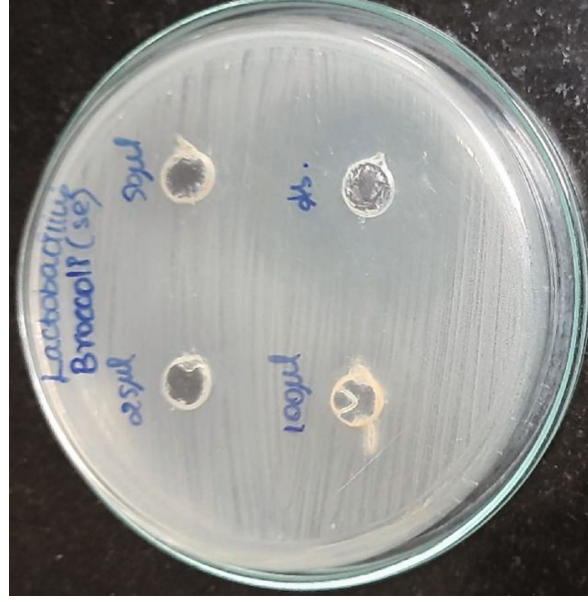

(b)

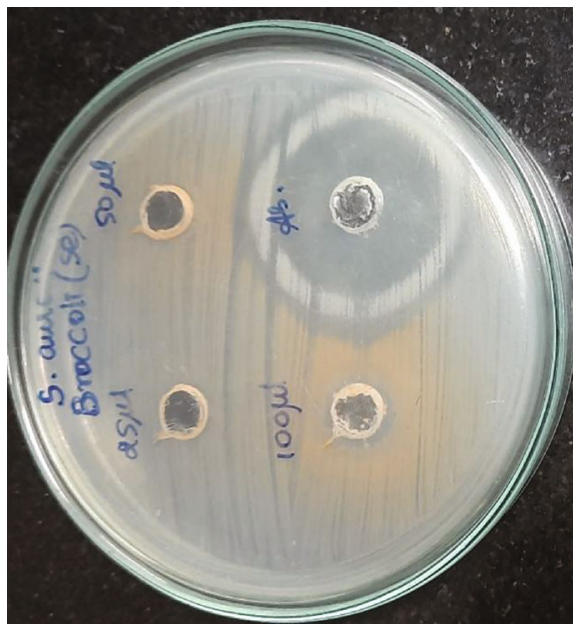

(d)

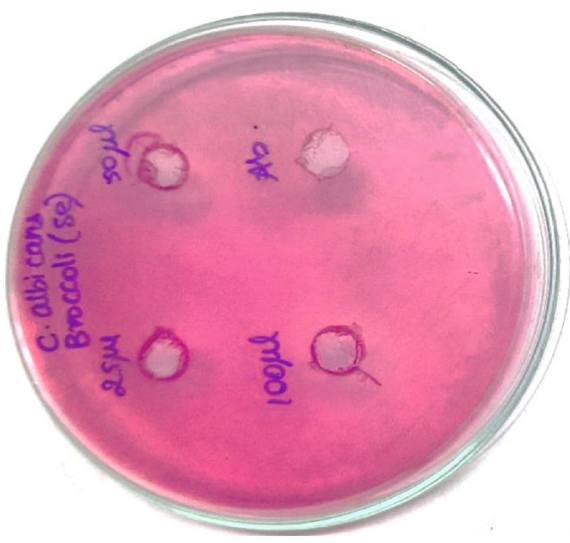

(e)

FIGURE 7: Anticariogenic activity of selenium nanoparticles: (a) S. mutans; (b) Lactobacillus sp.; (c) E. faecalis; (d) S. aureus; (e) C. albicans.

the reaction product. For synthesized extracts of SeNPs, the recorded EDaX showed a strong signal of $3 \mathrm{keV}$ selenium.

In our study, the bacterial growth was affected by the addition of various sodium selenite concentrations under aerobic conditions to the growth medium. Elevated concentrations of sodium selenite induce a lot of impairment to the genetic configuration in microbes. Thus, significant numbers of bacte- ria perish because of the stress caused by the existence of toxic inorganic compounds in the atmosphere, thereby reducing the production of selenium nanoparticles [8].

It has also been shown that selenium dioxide $\left(\mathrm{SeO}_{2}\right)$ as $\mathrm{Na}_{2} \mathrm{SeO}_{3}$ causes noxious effects on microbial plasmid DNA under stress conditions of $\mathrm{H}_{2} \mathrm{O}_{2}$, but with greater intensity, whereas other selenium-containing compounds such as 


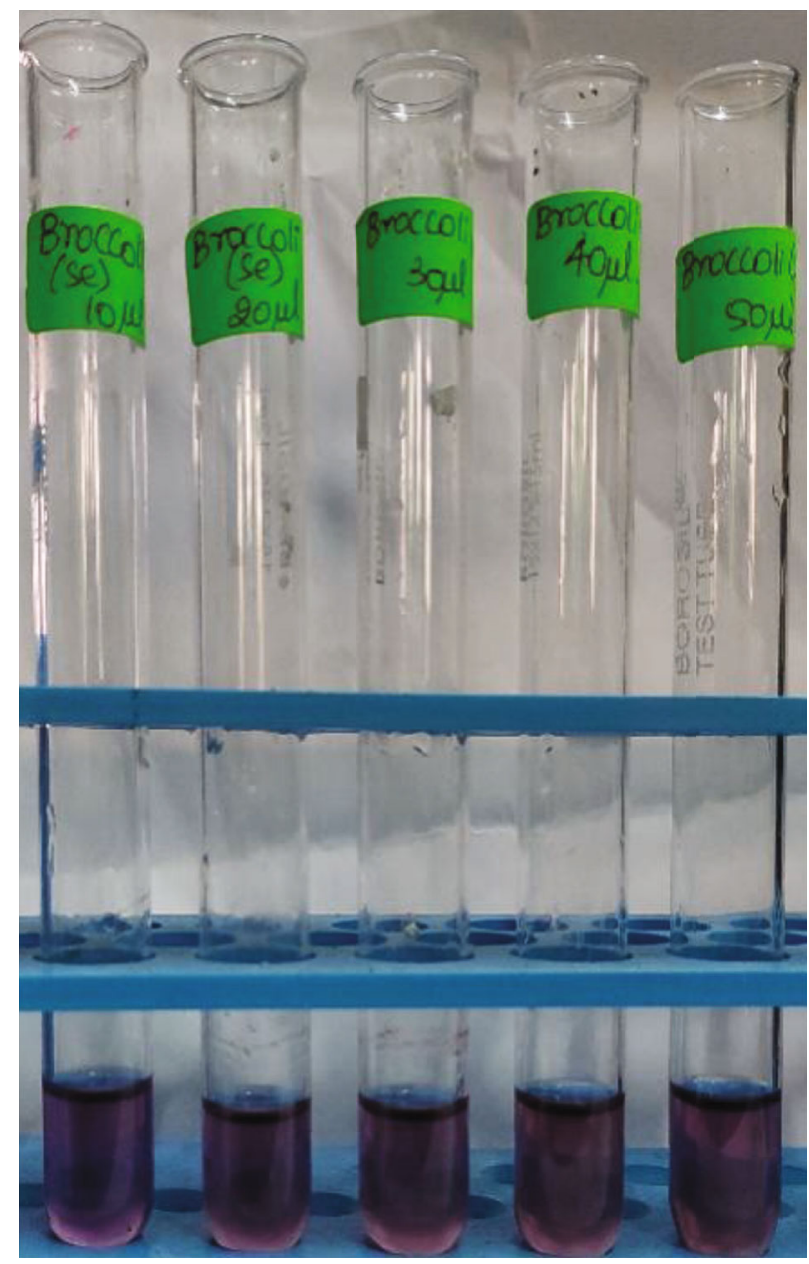

(a)

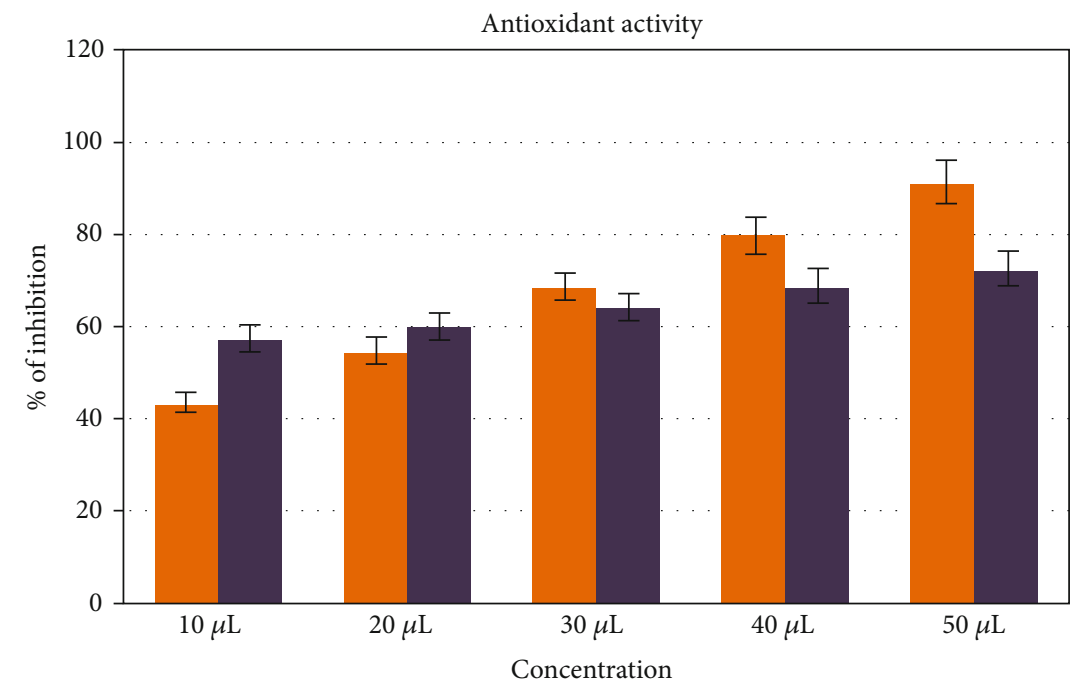

Standard

SeNPs

(b)

FIGURE 8: Antioxidant activity of selenium nanoparticles: (a) color changes; (b) graph representing the antioxidant activity at percentage of inhibition. 
$\mathrm{Na}_{2} \mathrm{SeO}_{4}$ and $\mathrm{Na}_{2} \mathrm{Se}$ have some inhibitory effects on Escherichia coli plasmid DNA. Previous studies support our study of the antibacterial activity of selenium on Staphylococcus and suggest that selenium is capable of preventing the development of biofilms by bacteria and cytotoxic effect has been demonstrated by SeNPs [28]. In live aureus assays, it was demonstrated that SeNPs inhibited pneumonia in cell lines [29]. In this analysis, the antimicrobial impact of SeNPs on microbes was observed.

The mechanism attributed may be owed to the interruption of the cell wall, indicating that the selenium nanoparticles were able to penetrate the cell wall. The antibacterial role of these selenium nanoparticles can be distinguished by morphological alterations in bacterial strains both intracellular and extracellular. The antimicrobial behavior of selenium nanoparticles is mainly due to the formation of reactive oxygen species, which contributes to the disruption of bilayer phospholipids where intracellular proteins are associated with and inactivated by SeNPs or where the sulfhydryl and the thiol groups existing in membrane proteins can react with and eventually denature them. By altering the cycle of protein synthesis, interfering with the mechanism of respiratory or food metabolism, or impairing the replication of DNA, oxidative stress caused by reactive oxygen induces cell death [30].

Toxicological examinations have demonstrated that small selenium nanoparticles possess a higher surface area and those particle numbers per unit mass can induce harmful respiratory damage and inflammation. The larger particles about $100 \mathrm{~nm}$ are more easily engulfed by macrophages. Their aggregation can, thus, decrease the toxicity of the nanoparticles [31,32].

Selenium nanoparticles can be used in various applications in the prevention of caries in dental tissues. Incorporating selenium nanoparticles in toothpaste, chewing gums, and mouthwashes can potentially help control the growth of caries causing microbes. Similarly, selenium nanoparticles can be incorporated in luting cement, endodontic sealers, pit and fissure sealants, and restorative cement to control secondary dental caries. Further extensive research in these domains will enhance our understanding of the therapeutic effectiveness of selenium nanoparticles in the prevention of dental caries.

\section{Conclusion}

Nanomaterials have emerged as promising therapeutic agents in the prevention and treatment of dental caries. In this study, SeNPs were synthesized using Brassica oleracea extract, characterized using UV-vis spectroscopy which confirmed the SeNP formation and electron microscopic images from TEM and SEM which showed nanoparticles less than $50 \mathrm{~nm}$, and evaluated for the antibacterial activity against dental caries-causing pathogens, and it was found that SeNPs showed effective antimicrobial activity against cariogenic pathogens. The SeNPs synthesized with Brassica oleracea extract can be incorporated in toothpastes, gums, and mouthwashes that are cost-effective and also biocompatible and effective for the prevention of dental caries.

\section{Data Availability}

The data used to support the findings of this study are included within the article.

\section{Conflicts of Interest}

The authors declare that there is no conflict of interest.

\section{Authors' Contributions}

DG and SR designed, carried out research, and wrote the manuscript.

\section{References}

[1] S. al-Musawi, S. Albukhaty, H. al-Karagoly et al., "Antibacterial activity of honey/chitosan nanofibers loaded with capsaicin and gold nanoparticles for wound dressing," Molecules, vol. 25, no. 20, article 4770, 2020.

[2] M. Jabir, U. I. Sahib, Z. Taqi et al., "Linalool-loaded glutathione-modified gold nanoparticles conjugated with CALNN peptide as apoptosis inducer and NF- $\kappa$ B translocation inhibitor in SKOV-3 cell line," International Journal of Nanomedicine, vol. Volume 15, pp. 9025-9047, 2020.

[3] Z. Qiao, Y. Xie, Y. Qian, and Y. Zhu, " $\gamma$-Irradiation preparation and characterization of nanocrystalline ZnS," Materials Chemistry and Physics, vol. 62, no. 1, pp. 88-90, 2000.

[4] S. Menon, H. Agarwal, S. Rajeshkumar, P. Jacquline Rosy, and V. K. Shanmugam, "Investigating the antimicrobial activities of the biosynthesized selenium nanoparticles and its statistical analysis," Bionanoscience, vol. 10, no. 1, pp. 122-135, 2020.

[5] P. Narasingarao and M. M. Häggblom, "Identification of anaerobic selenate-respiring bacteria from aquatic sediments," Applied and Environmental Microbiology, vol. 73, no. 11, pp. 3519-3527, 2007.

[6] J. Zhang, S.-Y. Zhang, J.-J. Xu, and H.-Y. Chen, "A new method for the synthesis of selenium nanoparticles and the application to construction of $\mathrm{H}_{2} \mathrm{O}_{2}$ biosensor," Chinese Chemical Letters, vol. 15, no. 11, pp. 1345-1348, 2004.

[7] P. A. Tran and T. J. Webster, "Antimicrobial selenium nanoparticle coatings on polymeric medical devices," Nanotechnology, vol. 24, no. 15, article 155101, 2013.

[8] N. Srivastava and M. Mukhopadhyay, "Biosynthesis and structural characterization of selenium nanoparticles mediated by Zooglea ramigera," Powder Technology, vol. 244, pp. 26-29, 2013.

[9] H. Wang, J. Zhang, and H. Yu, "Elemental selenium at nano size possesses lower toxicity without compromising the fundamental effect on selenoenzymes: comparison with selenomethionine in mice," Free Radical Biology \& Medicine, vol. 42, no. 10, pp. 1524-1533, 2007.

[10] A. G. al-Dulimi, A. Z. al-Saffar, G. M. Sulaiman et al., "Immobilization of 1-asparaginase on gold nanoparticles for novel drug delivery approach as anti-cancer agent against human breast carcinoma cells," Journal of Materials Research and Technology, vol. 9, no. 6, pp. 15394-15411, 2020.

[11] M. S. Jabir, Y. M. Saleh, G. M. Sulaiman et al., "Green synthesis of silver nanoparticles using Annona muricata extract as an inducer of apoptosis in cancer cells and inhibitor for NLRP3 inflammasome via enhanced autophagy," Nanomaterials, vol. 11, no. 2, p. 384, 2021. 
[12] O. al Rugaie, M. Jabir, R. Kadhim et al., "Gold nanoparticles and graphene oxide flakes synergistic partaking in cytosolic bactericidal augmentation: role of ROS and NOX2 activity," Microorganisms, vol. 9, no. 1, p. 101, 2021.

[13] G. M. Sulaiman, E. H. Ali, I. I. Jabbar, and A. H. Saleem, "Synthesis, characterization, antibacterial and cytotoxic effects of silver nanoparticles," Digest Journal of Nanomaterials and Biostructures, vol. 9, no. 2, pp. 787-796, 2014.

[14] W. J. al-Kaabi, S. Albukhaty, A. J. M. al-Fartosy et al., "Development of Inula graveolens (L.) plant extract electrospun/polycaprolactone nanofibers: a novel material for biomedical application," Applied Sciences, vol. 11, no. 2, p. 828, 2021.

[15] W. Zhang, Z. Chen, H. Liu, L. Zhang, P. Gao, and D. Li, “Biosynthesis and structural characteristics of selenium nanoparticles by Pseudomonas alcaliphila," Colloids and Surfaces. B, Biointerfaces, vol. 88, no. 1, pp. 196-201, 2011.

[16] C. I. R. Gill, "The effect of cruciferous and leguminous sprouts on genotoxicity, in vitro and in vivo," Cancer Epidemiology, Biomarkers \& Prevention, vol. 13, no. 7, pp. 1199-1205, 2004.

[17] G. Brandi, G. F. Schiavano, N. Zaffaroni et al., "Mechanisms of action and antiproliferative properties of Brassica oleracea juice in human breast cancer cell lines," The Journal of Nutrition, vol. 135, no. 6, pp. 1503-1509, 2005.

[18] E. H. Jeffery, A. F. Brown, A. C. Kurilich et al., "Variation in content of bioactive components in broccoli," Journal of Food Composition and Analysis, vol. 16, no. 3, pp. 323-330, 2003.

[19] R. A. Bagramian, F. Garcia-Godoy, and A. R. Volpe, "The global increase in dental caries. A pending public health crisis," American Journal of Dentistry, vol. 22, no. 1, pp. 3-8, 2009.

[20] R. H. Selwitz, A. I. Ismail, and N. B. Pitts, "Dental caries," The Lancet, vol. 369, no. 9555, pp. 51-59, 2007.

[21] T. Walsh, H. V. Worthington, A. M. Glenny et al., "Fluoride toothpastes of different concentrations for preventing dental caries in children and adolescents," Cochrane Database of Systematic Reviews, vol. 1, 2010.

[22] K. J. Toumba and M. E. J. Curzon, "A clinical trial of a slowreleasing fluoride device in children," Caries Research, vol. 39, no. 3, pp. 195-200, 2005.

[23] S. Maleki Dizaj, F. Lotfipour, M. Barzegar-Jalali, M.H. Zarrintan, and K. Adibkia, "Application of Box-Behnken design to prepare gentamicin-loaded calcium carbonate nanoparticles," Artificial Cells, Nanomedicine, and Biotechnology, vol. 44, no. 6, pp. 1475-1481, 2016.

[24] M. Hannig and C. Hannig, "Nanotechnology and its role in caries therapy," Advances in Dental Research, vol. 24, no. 2, pp. 53-57, 2012.

[25] L. Cheng, K. Zhang, M. D. Weir, M. A. S. Melo, X. Zhou, and H. H. K. Xu, "Nanotechnology strategies for antibacterial and remineralizing composites and adhesives to tackle dental caries," Nanomedicine, vol. 10, no. 4, pp. 627-641, 2015.

[26] M. A. S. Melo, S. F. F. Guedes, H. H. K. Xu, and L. K. A. Rodrigues, "Nanotechnology-based restorative materials for dental caries management," Trends in Biotechnology, vol. 31, no. 8, pp. 459-467, 2013.

[27] C. M. Debieux, E. J. Dridge, C. M. Mueller et al., "A bacterial process for selenium nanosphere assembly," Proceedings of the National Academy of Sciences of the United States of America, vol. 108, no. 33, pp. 13480-13485, 2011.

[28] S. Boroumand, M. Safari, E. Shaabani, M. Shirzad, and R. Faridi-Majidi, "Selenium nanoparticles: synthesis, characterization and study of their cytotoxicity, antioxidant and anti- bacterial activity," Materials Research Express, vol. 6, no. 8, article 0850d8, 2019.

[29] S. Shoeibi and M. Mashreghi, "Biosynthesis of selenium nanoparticles using Enterococcus faecalis and evaluation of their antibacterial activities," Journal of Trace Elements in Medicine and Biology, vol. 39, pp. 135-139, 2017.

[30] X. Chen, K. Cai, J. Fang et al., "Fabrication of seleniumdeposited and chitosan-coated titania nanotubes with anticancer and antibacterial properties," Colloids and Surfaces B, Biointerfaces, vol. 103, pp. 149-157, 2013.

[31] S. Chaudhary, P. Chauhan, R. Kumar, and K. K. Bhasin, "Toxicological responses of surfactant functionalized selenium nanoparticles: a quantitative multi-assay approach," Science of The Total Environment, vol. 643, pp. 1265-1277, 2018.

[32] R. Tarrahi, A. Khataee, A. Movafeghi, F. Rezanejad, and G. Gohari, "Toxicological implications of selenium nanoparticles with different coatings along with $\mathrm{Se}^{4+}$ on Lemna minor," Chemosphere, vol. 181, pp. 655-665, 2017. 